\title{
Aircraft wake vortex scenarios simulation package - WakeScene
}

\author{
Frank Holzäpfel ${ }^{\mathrm{a}, *}$, Michael Frech ${ }^{\mathrm{a}}$, Thomas Gerz ${ }^{\mathrm{a}}$, Arnold Tafferner ${ }^{\mathrm{a}}$, Klaus-Uwe Hahn ${ }^{\mathrm{b}}$, \\ Carsten Schwarz ${ }^{\mathrm{b}}$, Hans-Dieter Joos ${ }^{\mathrm{c}}$, Bernd Korn ${ }^{\mathrm{d}}$, Helge Lenz ${ }^{\mathrm{d}}$, Robert Luckner ${ }^{\mathrm{e}}$, \\ Gordon Höhne ${ }^{f}$ \\ a Institut für Physik der Atmosphäre, DLR-Oberpfaffenhofen, 82234 Weßling, Germany \\ b Institut für Flugsystemtechnik, DLR-Braunschweig, 38108 Braunschweig, Germany \\ c Institut für Robotik und Mechatronik, DLR-Oberpfaffenhofen, 82234 Weßling, Germany \\ d Institut für Flugführung, DLR-Braunschweig, 38108 Braunschweig, Germany \\ e Technische Universität Berlin, 10587 Berlin, Germany \\ f Airbus Deutschland GmbH, 21129 Hamburg, Germany
}

Received 26 March 2007; received in revised form 3 September 2007; accepted 3 September 2007

Available online 15 April 2008

\begin{abstract}
Wake-vortex advisory systems and modifications of ATC procedures that aim at increasing airport capacity without compromising safety have been developed in recent years. Prior to the introduction of such systems the associated risks must be assessed. The WakeScene (Wake Vortex Scenarios Simulation) Package allows to assess the encounter probability behind different wake-vortex generating aircraft during approach and landing. WakeScene consists of modules that model traffic mix, aircraft trajectories, meteorological conditions, wake vortex evolution, and potential hazard area. This manuscript introduces the operating sequence of WakeScene, the employed sub-models and data bases, the simulation environment and evaluation tools together with the so far accomplished validation work. Examples of WakeScene applications and an outlook on further developments of the software package conclude the report.
\end{abstract}

(c) 2008 Elsevier Masson SAS. All rights reserved.

\section{Zusammenfassung}

Konzeptionen von Wirbelschleppen-Warnsystemen und modifizierten Anflugverfahren zur Kapazitätssteigerung großer Flughäfen haben mittlererweile einen beachtlichen Reifegrad erreicht. Bevor derartige Systeme jedoch operationell genutzt werden können, müssen die mit ihrer Einführung verbundenen Risiken umfassend untersucht und bewertet werden. Das vorgestellte WakeScene (Wake Vortex Scenarios Simulation) Software Paket dient der Bestimmung der Wahrscheinlichkeit einer Wirbelschleppen Begegnung im Landeanflug für unterschiedliche Szenarien. WakeScene besteht aus mehreren Modulen, die den Verkehrsmix, die Flugzeugtrajektorien, die meteorologischen Bedingungen, das Wirbelschleppen Verhalten sowie die potenziellen Gefährdungsräume simulieren. Das vorliegende Manuskript beschreibt die verwendeten Modelle und Datenbasen, das Zusammenspiel der einzelnen Module, die Simulationsumgebung und Auswertewerkzeuge, sowie den Stand der Validierung der einzelnen Komponenten. Anwendungsbeispiele und ein Ausblick auf die geplanten Weiterentwicklungen des Software Pakets beschließen den Bericht.

(C) 2008 Elsevier Masson SAS. All rights reserved.

Keywords: Wake vortices; Risk assessment; Glide path adherence; Meteorological conditions

Schlüsselwörter: Wirbelschleppen; Sicherheitsanalyse; Gleitpfadabweichungen; Meteorologische Bedingungen

\footnotetext{
* Corresponding author. Tel.: +49 8153 282529; fax: +49 8153281841.

E-mail address: frank.holzaepfel@dlr.de (F. Holzäpfel).
}

\section{Introduction}

Aircraft generated wake vortices pose a potential risk to following aircraft in various flight phases. This applies to take-off and climb, to cruise during climb phases and descent phases, 
but also during following or crossing on the same altitude or at reduced vertical separation between flight levels (RVSM). A particular risk is given during approach and landing, because, on one hand, the aircraft density is increased along the glide path, and, on the other hand, wake vortices may stall or rebound in ground proximity [19]. These factors lead to the largest probability to encounter wake vortices during final approach $[3,4,30]$. Five years of UK wake encounter data indicate that more than one quarter of incident reports are from wake encounters at altitudes less than $500 \mathrm{ft}$ (see [30] part II, Section 8.2). Moreover, the pilot's possibility to counterbalance vortex encounters at low altitudes is substantially restricted.

The established wake-vortex aircraft separation standards [20] increasingly degrade aviation efficiency when traffic congestion limits airport capacity during landing and take-off. The most rapid growth scenario within a Eurocontrol study [1] indicates that in the year 2025 sixty European airports could be congested and as a result 3.7 million flights per year could not be met.

In recent years, wake-vortex advisory systems [11,30] (WVAS) and modifications of ATC procedures [30] have been developed that are meant to increase airport capacity while keeping safety at least at the existing level. However, prior to the introduction of any WVAS, the associated risks must be assessed as required by the Eurocontrol Safety Regulatory Requirement (ESARR 4) [7]. Any simulation model that shall be employed to prove the safety of a WVAS must constitute a sufficiently accurate representation of the projected operation and must be able to estimate the associated risk level.

For our purposes here these requirements can be translated into two major questions: (i) What is the probability to encounter vortices of a preceding aircraft during approach and landing? (ii) How hazardous is an encounter when it happens? To answer these questions we went through two phases: (1) identification of all relevant processes and (2) definition of the appropriate degree of details with which the relevant processes have to be modelled. Finally, it must be demonstrated that the developed tool fulfils the requirements (1) and (2).

The WakeScene (Wake Vortex Scenarios Simulation) Package, which is described in this manuscript, allows to assess the relative encounter probability behind different wake vortex generating aircraft within a domain ranging from the final approach fix to threshold. In cases with potential wake encounters all relevant parameters can be provided to VESA (Vortex Encounter Severity Assessment) $[14,24]$ which may subsequently perform detailed investigations of the severity of the encounter. WakeScene consists of elements that model traffic mix, aircraft trajectories, meteorological conditions, wake vortex evolution, and potential hazard area. The process and data flows are controlled and evaluated by the MATLAB-based environment MOPS (Multi Objective Parameter Synthesis) [21]. Other applications of WakeScene comprise the adjustment and optimisation of parameters within a WVAS and the estimation of its potential capacity gains. It may also be extended to related applications, for example the evaluation of wake vortex risks introduced by noise abatement procedures.
Other models intended for similar purposes are (1) WAVIR (Wake Vortex Induced Risk) [28] which has been developed by NLR and is capable to estimate frequencies of certain risk events in a given scenario. (2) ASAT (Airspace Simulation and Analysis for TERPS where TERPS stands for Terminal Instrument Procedures) devised by the Federal Aviation Administration and the Air Traffic Simulation Inc is a multifaceted computer tool for aviation related simulations and safety evaluations which has not been specifically designed as a wake vortex risk assessment model. Similar to WakeScene, ASAT has an interface to VESA that permits subsequent wake vortex encounter severity assessment. (3) The Vortex Risk Analysis Tool which has been developed by Det Norske Veritas with DLR expertise has been employed for the risk assessment of the High Approach Landing System/Dual Threshold Operation (HALS/DTOP) implemented at Frankfurt airport. HALS/DTOP aims at increasing the capacity of the closely spaced parallel runway system by employing a second threshold displaced by 1500 meters for the southern runway. (4) A comprehensive air traffic control wake vortex safety and capacity integrated platform has also been generated in the EU project ATC-Wake [29]. It should further be referred to [31] which focuses on the computational efficiency of the evaluation of rare events such as serious encounters by combining probability distributions of relevant parameters. The Wake Vortex Research Needs Report compiled by WakeNet2-Europe partners [30] provides a comprehensive survey on operational concepts designated to increase airport capacity and the regulatory framework which is relevant for the associated risk assessments as well as many other wake vortex related issues.

In the following sections first a survey on the operating sequence of WakeScene is given, then the employed sub-models, data bases, and simulation environment are described, followed by examples of the application of the software package, and an outlook on further developments.

\section{Survey on operating sequence}

WakeScene models final approaches to a single runway within 25 gates starting at the final approach fix which is situated $11 \mathrm{NM}$ before threshold (see Fig. 1). Along the final nautical mile the gates are separated by $1 / 6 \mathrm{NM}$ to properly resolve the interaction of wake vortices with the ground whereas at higher altitudes the gates are separated by $1 / 2 \mathrm{NM}$. The flowchart depicted in Fig. 2 sketches the operating sequence of WakeScene. Via simulation control (MOPS) the types of the generator aircraft and follower aircraft are selected. The Aircraft-Speed Model provides time, speed, and mass of generator and follower aircraft at different gate positions along the glide path. From this the Flight-Path Deviation Model computes random deviations from nominal glide path for generator and follower aircraft at all gates. The three modules Meteorological Data Base, Wake-Vortex Model, and Hazard-Area Model are then run in a loop over the 25 gates. Based on vertical profiles of wind speed and direction, air density, virtual potential temperature, turbulent kinetic energy, and eddy dissipation rate (Meteorological Data Base) and air- 


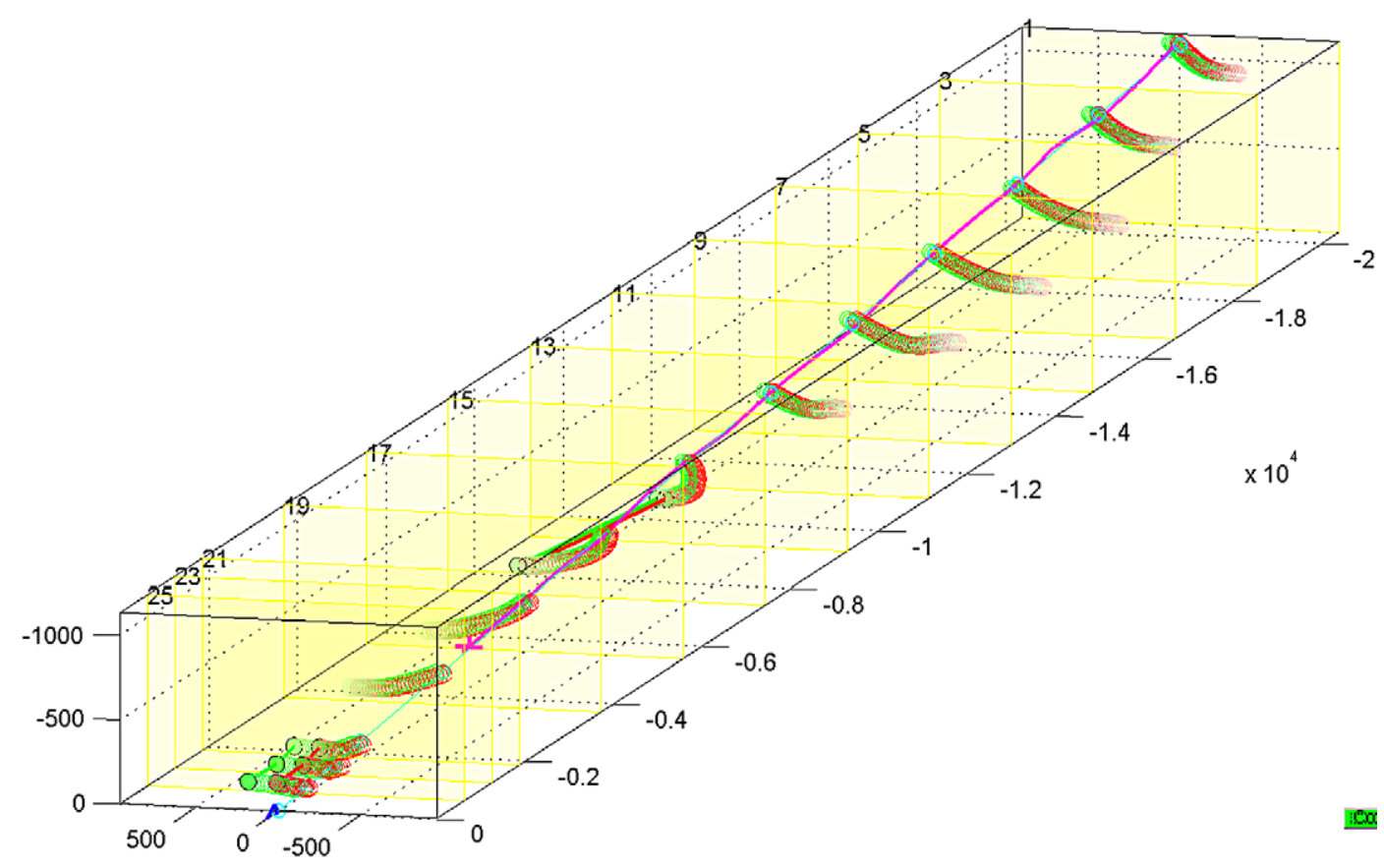

Fig. 1. Development of potential hazard areas of wake vortices visualised by circles in 13 gates from final approach fix (gate 1) to threshold (gate 25). Dimensions in meters. Wake-generating aircraft (heavy) has already landed, follower aircraft (medium, magenta) at about 2.5 NM before touch-down. Due to changes of wind directions wake vortices are transported in opposite directions at different heights.

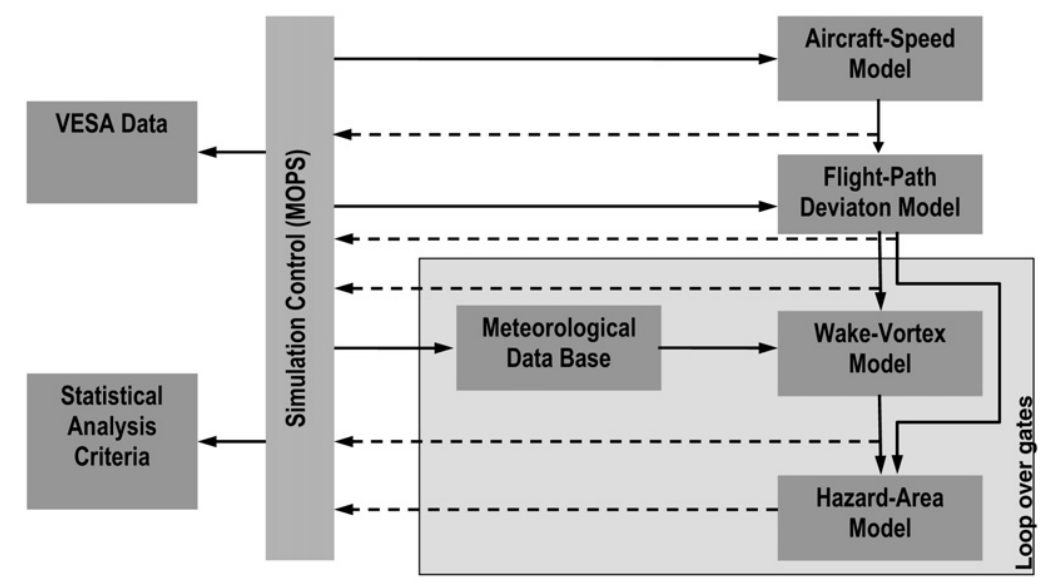

Fig. 2. WakeScene flowchart. Solid lines denote the data flow whereas dashed lines indicate the access of simulation control to data fluxes.

craft position, speed, mass, and span (Flight-Path Deviation Model) at one gate, the Wake-Vortex Model simulates the development of wake vortex trajectories, circulation, vortex core radius, and attitude of wake vortex axes. The Hazard-Area Model computes the distance between wake vortex and follower aircraft within each gate and supplies vortex and aircraft data to MOPS. From all these data MOPS computes defined criteria, like minimal distance between wake vortex and follower aircraft and the respective vortex circulation and height, which are interpolated between the gates and statistically analysed. Finally, data needed for further investigations with VESA are deduced and stored. The results are optionally visualised in graphs of the statistics, 2D and 3D views (see Fig. 1) or animations of the approaches of subsequent aircraft.

\section{Meteorological data base}

It is generally accepted that wake vortex behaviour is largely controlled by environmental parameters $[11,13,16]$. The variety of parameter combinations observed in the planetary boundary layer and their transformation on wake vortex behaviour lead to a significant manifold of situations which is not likely to be covered with simple methods. Therefore, for WakeScene significant effort has been put in the provision of a realistic and comprehensive meteorological data base.

\subsection{Production and description of data base}

A one-year simulation of realistic meteorological conditions has been produced for the Frankfurt terminal area with 
the non-hydrostatic mesoscale weather forecast model system NOWVIV (NOwcasting Wake Vortex Impact Variables [11]). NOWVIV has been successfully employed for predictions of wake vortex environmental parameters in the field campaigns WakeOP 2001 [17] and WakeTOUL 2002 [18] of projects Wirbelschleppe and C-Wake, in the first flight test campaign 2003 of AWIATOR [18], in the measurement campaign at Frankfurt airport accomplished in fall 2004 [19], and during the demonstration of the DLR Wake-Vortex Prediction and Monitoring System at Frankfurt airport in winter 2006/2007. NOWVIV comprises a full physics package including boundary layer turbulence, surface energy and momentum balance, soil physics, radiation processes including cloud effects, cumulus convection, and cloud physics. The core of NOWVIV is the mesoscale model MM5 [12] where a Yamada \& Mellor 2.5 level turbulence closure scheme is employed from which turbulent kinetic energy (TKE) is computed as a prognostic variable. The eddy dissipation rate is extracted from the TKE budget equation.

For the current purpose NOWVIV was adapted to the Frankfurt airport area. Two nested domains with sizes of about $250 \times 250 \mathrm{~km}^{2}$ and about $90 \times 90 \mathrm{~km}^{2}$ centred on Frankfurt airport with grid distances of $6.3 \mathrm{~km}$ and $2.1 \mathrm{~km}$, respectively, were used. The model employs 60 vertical levels such that in the altitude range of interest $(z<1100 \mathrm{~m}$ above ground) 26 levels yield a vertical resolution varying between $8 \mathrm{~m}$ and $50 \mathrm{~m}$. Initial and boundary data were taken from the numerical data assimilation model LM (Local Model, [5]) of DWD (German Weather Service). These data represent the best possible forcing of NOWVIV since actual observations (radio soundings, AMDAR (Aircraft Meteorological Data Relay), satellite data, surface observations, etc.) are used to analyse the state of the atmosphere. Detailed topography, land use and soil type data for the Frankfurt area were employed.

Profiles of meteorological data were extracted at 25 locations separated by one nautical mile along the glide paths for approaches on the 07 and 25 runways. An output frequency of 10 minutes was selected. The resulting number of profiles amounts to about $1.3 \times 10^{6}$. The meteorological quantities comprise the three wind components, air density, virtual potential temperature, turbulent kinetic energy, eddy dissipation rate (EDR), and pressure.

\subsection{Validation of meteorological data base}

The 1-year meteorological data base has been validated against a 30-year wind climatology and a 40-days subset has been compared to ultrasonic anemometer, SODAR/RASS, and lidar measurement data acquired at Frankfurt airport at height levels ranging from the surface up to $300 \mathrm{~m}$ (for details see [10]). Case studies with weak and strong synoptic forcing complement the assessment. Assessments of wake prediction skill based on predictions of meteorological conditions with NOWVIV can be found in $[9,17]$.

Exemplarily for the verification work, Fig. 3 shows the comparison of the 1-year synthetic wind data with the 30-year surface wind climatology. The climatology considers winds av- eraged over one hour measured at $10 \mathrm{~m}$ above ground in a time frame from 1967 to 1997 . The comparison assumes that the 1-year representation of daily weather is already close to climatological conditions at the airport. The observed main surface wind directions are not only the result of predominant synoptic patterns, but are also influenced by the orography in the vicinity of the airport, here in particular the Taunus mountain ridge.

The joint frequency distribution of wind speed and direction established with NOWVIV (upper panel) shows in general good agreement with the climatology (lower panel). The Frankfurt wind climatology is characterised by two main wind directions: South-westerly winds with a peak around $200 \mathrm{deg}$ and north-easterly winds around $50 \mathrm{deg}$. For both main wind directions the corresponding peak in the mean wind speed is between 2-4 m/s. In the synthetic data the occurrence of stronger winds is slightly under-represented. Part of the minor differences in wind direction can be attributed to climate variability and trends. For example, in accordance with NOWVIV predictions, a higher frequency of easterly winds is noted by controllers at Frankfurt airport in recent years with more frequent landings on runway 07.

\section{Aircraft-speed model}

The risk to encounter a wake vortex is strongly correlated with the actual flight paths of the vortex generating aircraft and the encountering aircraft in space and time. The Aircraft-Speed Model employs a flight management system that defines the approach speed schedule and provides speed and mass of generator and follower aircraft at the different gate positions. The Flight-Path Deviation Model (next section) generates stochastic vertical and lateral deviations from the nominal glide path within the respective gates.

Currently, WakeScene leaves the choice between four heavy weight-class aircraft as wake generators and two medium weight-class follower aircraft. The Aircraft-Speed Model considers the aerodynamics and flight mechanics of the respective aircraft types and allows for variations in aircraft weight and speed. The calculations of speed profiles are based on realistic aircraft simulations using the BADA (Base of Aircraft Data) [6] flight model of EUROCONTROL taking into account the actual weight and aircraft configuration (flaps and gear setting). The speed profile is determined by simulating the behaviour of an autopilot following the ILS glide slope and localiser.

The trajectories of the aircraft pairing are calculated independently and their starting times are adjusted such that the ICAO separations between leading and following aircraft are guaranteed at the threshold. Accelerations and decelerations are deduced from a balance of lift, drag, and thrust, where it is assumed that the required lift equals the actual weight. The drag for the actual configuration, thrust maxima and minima and the fuel consumption are computed for each aircraft employing the BADA database. With low pass filters for the rate of thrust change and flight path angle change a realistic trajectory can be calculated. This has been demonstrated in various flight simulator investigations and flight trials with the DLR 

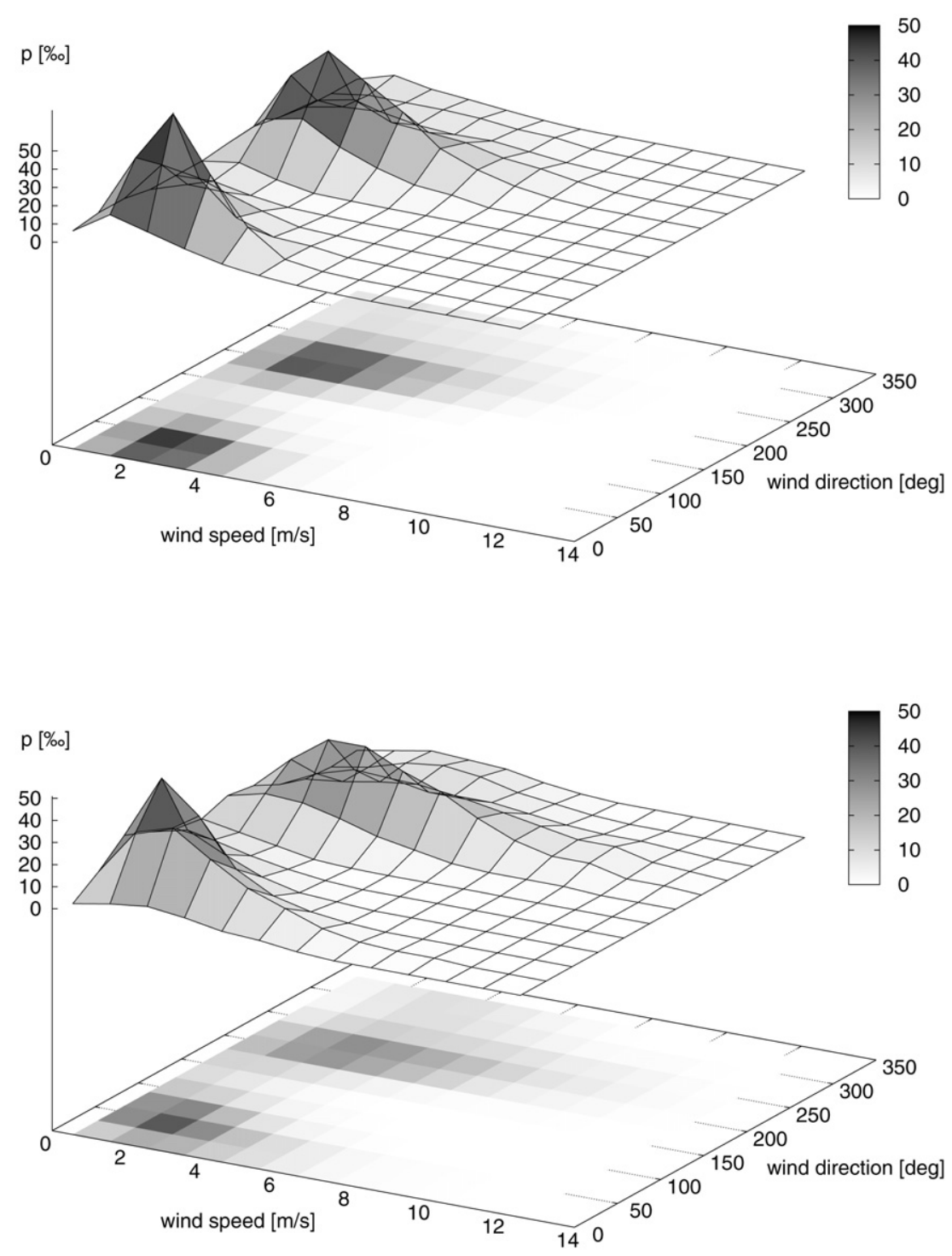

Fig. 3. Joint frequency distribution of wind speed and direction (in per mil) based on the 1-year NOWVIV run, upper panel, and the Frankfurt airport 30-year climatology, lower panel.

research aircraft ATTAS (Advanced Technology and Testing Aircraft System) [22].

\section{Flight-path deviation model}

The Flight-Path Deviation Model predicts stochastic spatial deviations from the nominal glide path from final approach fix to touchdown. Nominal trajectories depend on operational procedures defined by international standard which in turn are based on the available hardware for navigation services. The related requirements obey operational needs and safety aspects. Actual flight path deviations from nominal trajectories depend on the aircraft itself (aircraft dynamics), external influences (atmospheric disturbances), and the precision of the control system (autopilot/pilot skill).

The Flight-Path Deviation Model employs real flight path deviation data collected within the FLIP study [8], an investigation of the navigational performance of ILS (Instrument Landing System) approaches at Frankfurt airport. The FLIP study provides statistics of 35,691 tracks of precision approaches on Frankfurt ILS of runways 25L/R. It does not differentiate between manual and automatic approaches. The study indicates that the measured flight path deviations are much smaller than specified by ICAO localiser and glide slope tolerances.

The Flight-Path Deviation Model assumes that the distances between the control gates along the approach corridor are not too short to allow for independent treatment of the statistics at each gate, i.e. the aircraft is able to fly from the position calculated at one gate to the position calculated at the next gate without unrealistic manoeuvres. It was not investigated to which extent this independence assumption is justified. Fig. 4 shows vertical stochastic deviations at 25 gates along the approach path for one aircraft pairing which are produced employing vertical standard deviations of glide path adherence found in the FLIP study and a random number generator. The same procedure is applied for lateral deviations. 


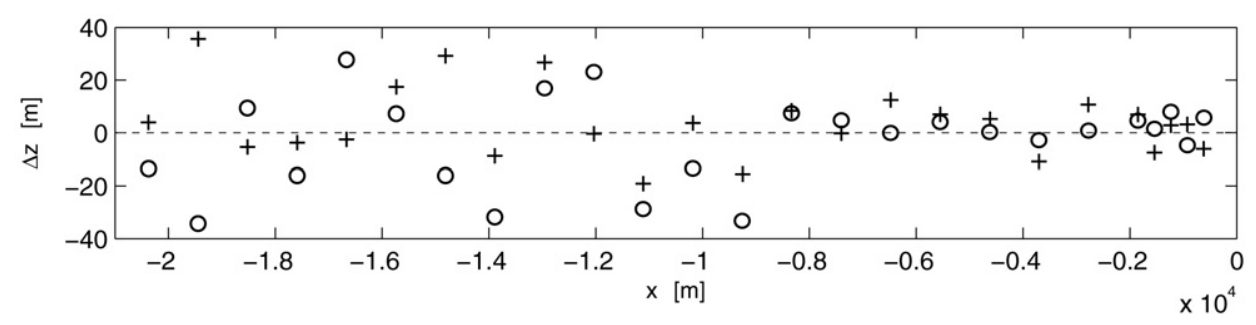

Fig. 4. Vertical deviations of generator aircraft (o) and follower aircraft (+) from nominal approach path in the 25 gates.
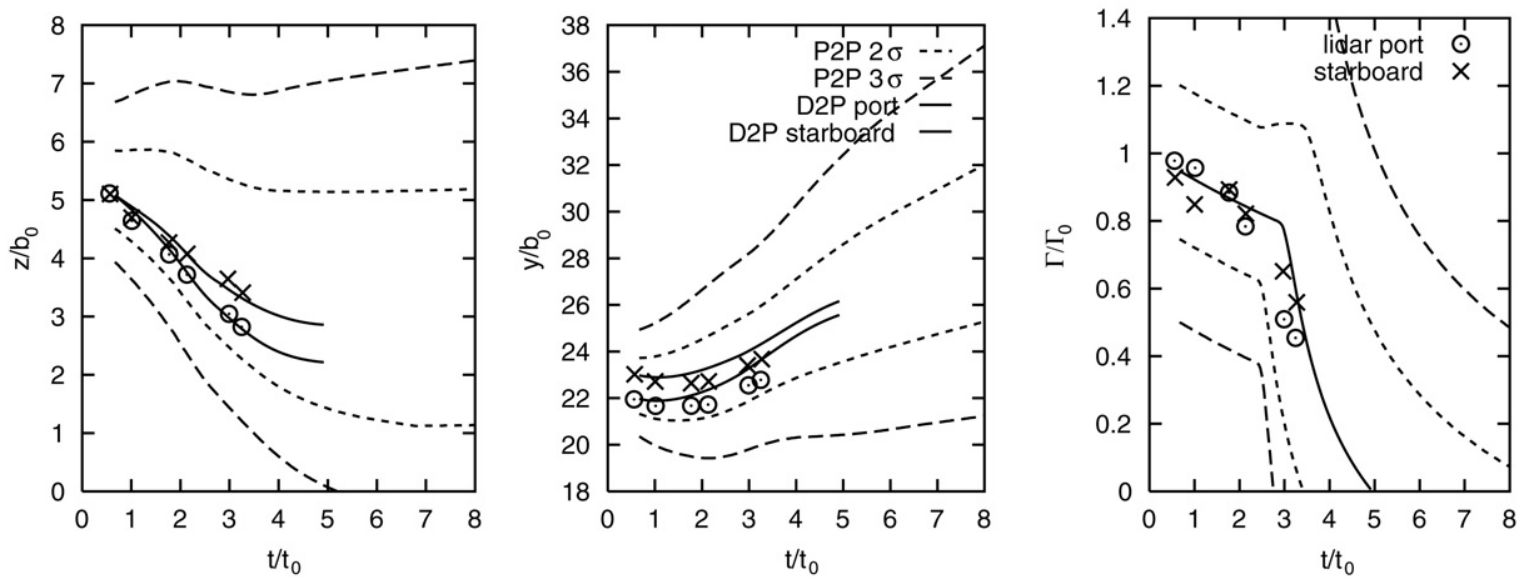

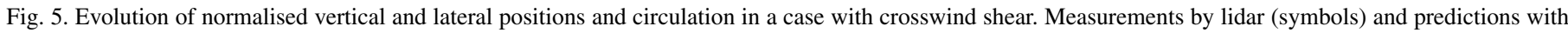

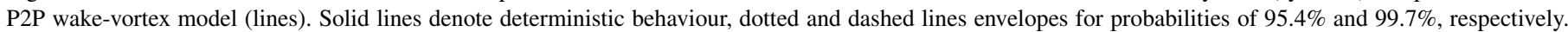
Normalisation of parameters based on initial values of vortex spacing, circulation, and time needed to descend one vortex spacing.

\section{Wake-vortex prediction models}

WakeScene provides a choice between different parametric wake-vortex prediction models. These are D2P and S2P, a deterministic and a stochastic version of the Probabilistic Two-Phase wake-vortex decay model (P2P) and APA, the AVOSS Prediction Algorithm [25,26]. Because the APA is merely used for reference purposes it is not further described here.

The P2P, which constitutes the basis of its deterministic and stochastic versions D2P (used by default) and S2P, is described in detail in [15]. Applications, assessments and further developments are reported in [17-19]. P2P accounts for the effects of wind, axial- and crosswind shear, turbulence, stable thermal stratification, and ground proximity.

The model equations are derived from the analytical solution of the spatiotemporal circulation evolution of the decaying potential vortex and are adapted to wake vortex behaviour as observed in large eddy simulations and experiments. Vortex decay progresses in two phases, a diffusion phase followed by rapid decay (see Fig. 5). The model allows for stagnating or even rebounding vortices with non-zero circulation in strongly stably stratified environments and pronounced wind shear situations.

Precise deterministic wake vortex predictions are not feasible operationally. Primarily, it is the nature of turbulence that deforms and transports the vortices in a stochastic way and leads to considerable spatiotemporal variations of vortex position and strength. Moreover, uncertainties of aircraft parameters and the variability of environmental conditions must be taken into account. Therefore, the output of P2P consists of confidence intervals for vortex position and strength (see Fig. 5). The confidence intervals can be adjusted to defined degrees of probability which are derived from statistics of measured wake vortex behaviour [18]. In total, P2P has been validated against data of over 1300 cases gathered in two US and four European measurement campaigns.

The deterministic version of $\mathrm{P} 2 \mathrm{P}$, termed $\mathrm{D} 2 \mathrm{P}$, provides a single temporal sequence of intermediate vortex positions and strengths per run (see solid lines in Fig. 5). The stochastic model version S2P provides stochastic realisations of vortex predictions which are randomly selected from probability density distributions predicted by P2P [18].

Further output provided to VESA includes vortex core radii and the interception angles between aircraft flight path and vortex axis, the so-called encounter angles. A simple vortex-core parameterisation is derived from the core radius growth of the decaying potential vortex which is adapted to core size measurement data and the two-phase concept of P2P.

Encounter severity strongly depends on encounter angles. To parameterise wake vortex attitude angles large eddy simulation data comprising the evolution of turbulent wake vortices in quiescent, turbulent, and convective atmospheres are analysed regarding inclination angles, azimuth angles, and bank angles of wake vortices. Statistics of local wake vortex angles are determined as functions of local circulation. Stochastic wake vortex angles are predicted based on joint probability density distri- 


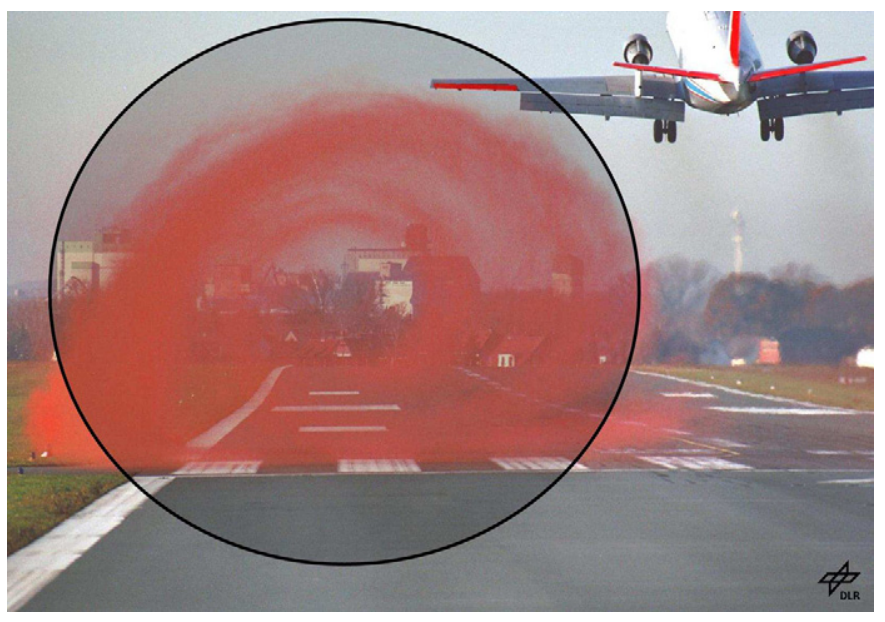

Fig. 6. Area of Interest centred on wake vortex axis. Wake-vortex in ground proximity generated by ATTAS research aircraft visualised by red smoke.

butions of wake vortex angles and vortex circulation. The final interception angles between aircraft flight path and vortex axis result from the combination of wake vortex angles with the attitude of flight of the follower aircraft.

\section{Hazard-Area model}

The Hazard-Area Module computes whether the trailing aircraft penetrates a circular Area of Interest around the vortex axis (see Fig. 6). The dimensions of this area are adjusted such that all cases where the aircraft passes the vortex axes in larger distances than the clearance distance (radius of Area of Interest) are assumed to be definitely uncritical. If the distance between the follower aircraft (represented by its centre of gravity) and one of the vortex axes falls short below the clearance distance, VESA (see next section) will compute encounter severity. To diagnose the closest approach of the follower aircraft to wake vortex centres, WakeScene interpolates aircraft trajectories and wake trajectories between the gates.

\section{Vortex Encounter Severity Assessment}

VESA (Vortex Encounter Severity Assessment) [14,24] is a separate software package developed by Airbus to determine the severity of wake encounters based on six-degrees-offreedom flight simulations. The variables provided to VESA comprise vortex parameters (position, attitude, and strength), configuration and flight attitude of generator and follower aircraft, as well as meteorological data. VESA includes wellestablished wake-vortex velocity models and a dedicated aerodynamic strip model to compute the wake-induced forces and moments acting on the wake encountering aircraft. These forces and moments are the input to the flight simulation of the follower aircraft. Depending on the application of the encounter simulation, it is controlled by an autopilot, a pilot model or by a pilot in simulator tests. For landing the pilot model consists of three modules: a module that simulates the behaviour of the pilot during ILS tracking, a module that takes into account the behaviour of the pilot during recovery from the encounter, and a module that triggers the initiation of a go-around in case certain severity limits are exceeded. Finally, the vortex-induced aircraft responses are assessed with the help of severity criteria that correlate the objective aircraft parameters with the subjective assessments of pilots known from piloted simulator tests.

The probability of severe encounters is equal to the encounter probability which is determined by WakeScene times the probability to exceed severity limits which is computed by VESA.

\section{Simulation control and evaluation}

The process and data flows are controlled and evaluated by the MATLAB-based environment MOPS (Multi Objective Parameter Synthesis) [21]. MOPS supports Monte-Carlo Simulation as well as dedicated parameters studies. A control panel allows for convenient control and evaluation of the runs. It enables to adjust the stochastically varied parameters like aircraft weight and speed and to select aircraft types or wake vortex prediction models. It is also possible to configure date and time intervals within the meteorological data base.

MOPS supports different interpolation schemes (linear used by default and different splines) between the gates to evaluate the minimal distance between wake vortex axes and the centre of gravity of the follower aircraft in planes aligned parallel to the gates. For the thus identified vortex segment further criteria like the respective vortex circulation, age, altitude, and axial position are determined. All criteria are statistically analysed. Search in the simulation data for the identification of critical cases is possible both numerically and based on a graphical approach. Finally, data needed for further investigations with VESA are deduced and stored. The results are optionally visualised in graphs of vortex evolutions of individual flights, statistics of various criteria (see Fig. 7), 2D and 3D views (see Fig. 1) or animations of the approaches of subsequent aircraft.

\section{Application}

As an example for applications of the WakeScene package we present statistics from a Monte-Carlo simulation with a sample size of 100,000 approaches of heavy aircraft (B747400) followed by medium (A320) and light medium aircraft (VFW614) which obey the 5 NM ICAO separation. We employ meteorological data of the full one-year data base and wake vortex predictions with the D2P model.

Fig. 7 displays histograms of follower aircraft altitude, distance to the wake vortex, vortex age, vortex circulation, and vortex separation where all parameters are evaluated for the instant of the closest approach (CA) of follower aircraft and trailing vortices. The frequencies of these five parameters are plotted independently, that is a correlation between e.g. the CA and vortex strength can not be inferred. The Area of Interest is not considered for the compilation of the histograms.

Fig. 7a shows that $64 \%$ of the CAs occur below an altitude of $300 \mathrm{ft}$ above ground (first three bins). Within this altitude range clearance of the flight corridor by descent and advection of the vortices is restricted: stalling or rebounding vortices may 

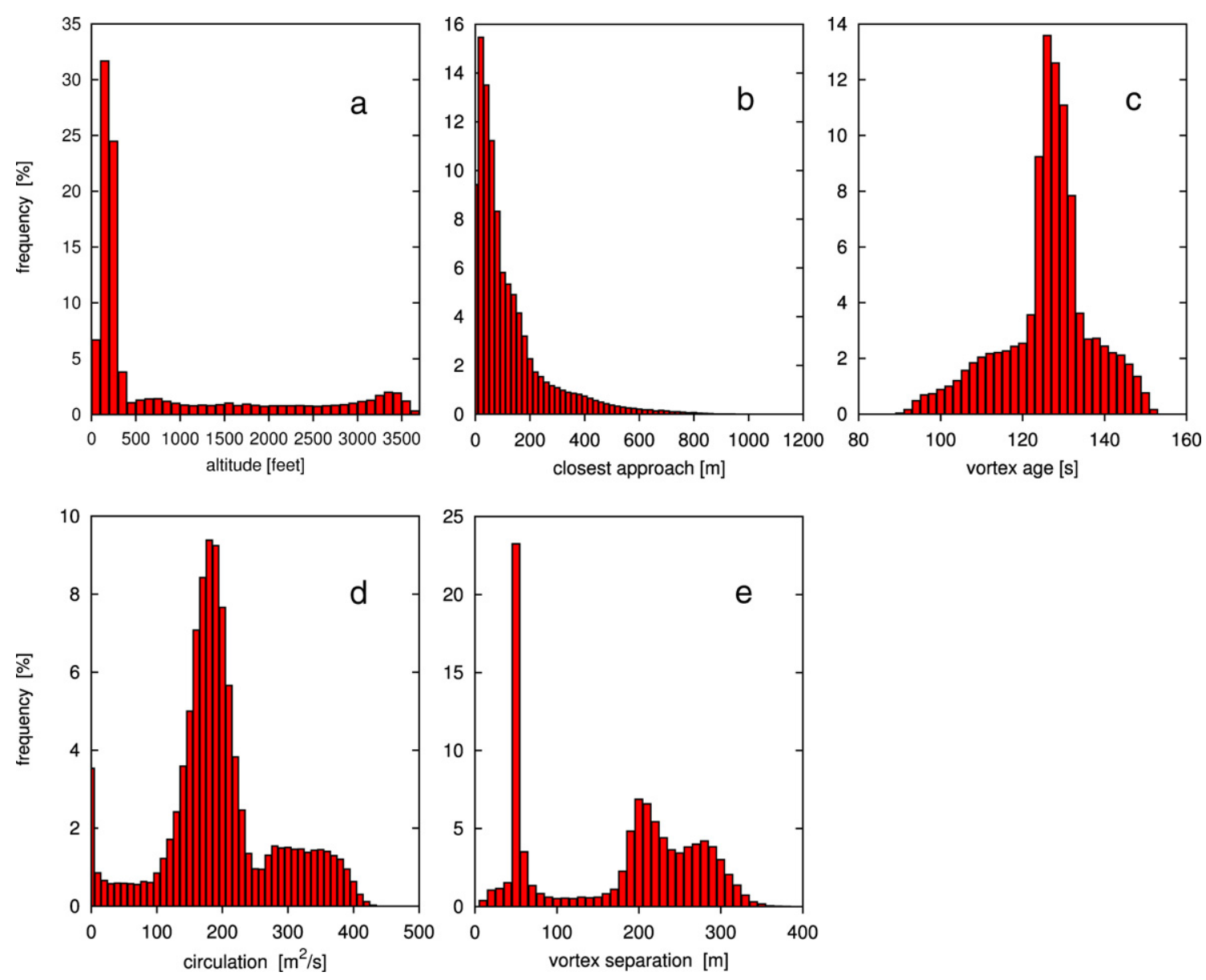

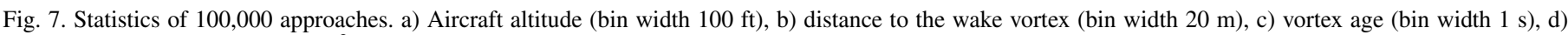

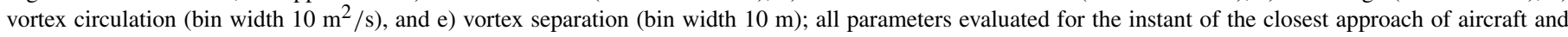
vortex.

not clear the flight path vertically and weak crosswinds may be compensated by vortex-induced lateral transport. The minor peak around $3400 \mathrm{ft}$ develops in runs where the leading aircraft employs sufficiently higher approach speeds. As a consequence the temporal separation between subsequent aircraft increases in time and the slower follower aircraft encounters the youngest wake vortices at the highest gates. Fig. $7 \mathrm{~b}$ reveals that the CAs amount to less than $30 \mathrm{~m}$ in $25 \%$ of the cases whereas a few cases exist with a CA beyond $1 \mathrm{~km}$. The median of the CAs is $71 \mathrm{~m}$. Fig. 7c, $d$ indicate a considerable range of vortex ages between 90 and $153 \mathrm{~s}$ corresponding to vortex strengths between 0 and $430 \mathrm{~m}^{2} / \mathrm{s}$. Only about $3.5 \%$ of vortices have completely decayed at the instant of the CA. The frequency of CAs where the wake vortices still have a circulation above $100 \mathrm{~m}^{2} / \mathrm{s}$ during a CA closer than $10 \mathrm{~m}$ amounts to $8 \%$. For wake vortices generated by an A300-600 at the lower end of the heavy weight class, this probability drops to zero. A clear indication that considerable capacity gains could be achieved by simply increasing the number of aircraft weight class categories. Fig. 7e illustrates that in $23 \%$ of the CAs the vortices still approximately retain their initial vortex spacing of $50.6 \mathrm{~m}$. The cluster of vortex separations beyond $180 \mathrm{~m}$ represents the separation range typically occurring after vortex rebound in ground proximity, whereas vortex separations around $50 \mathrm{~m}$ correlate with cross- wind shear situations where vortex tilting and the connected crosswind advection may cause either an increase or decrease of vortex spacings. Note that the characteristics of the described distributions are already fully met with a sample size of only 10,000 approaches.

Fig. 8 displays encounter frequencies dependent on the distance to the vortex and the respective circulation, $\Gamma$, for encounters within the complete considered altitude range (left) and below $300 \mathrm{ft}$ (right). The comparison of the left and right figure indicates that the majority of encounters occur in ground proximity (e.g. $95 \%$ for $\Gamma>150 \mathrm{~m}^{2} / \mathrm{s}$ ), whereas the more severe and infrequent encounters with $\Gamma>300 \mathrm{~m}^{2} / \mathrm{s}$ only occur at altitudes above $300 \mathrm{ft}$. Obviously, the interaction with the ground ensures vortex decay rates that prevent very strong encounters at low altitudes whereas atmospheric conditions aloft sometimes allow for longer living vortices which enable stronger encounters [19].

The fact that only a single encounter with $\Gamma>400 \mathrm{~m}^{2} / \mathrm{s}$ arises from the simulated 100,000 approaches (filled circle in Fig. 8 left) demonstrates that WakeScene is capable of reproducing very rare events. The underlying parameters of this severe encounter are: Nine nautical miles before touchdown the aircraft pair is only separated by $100 \mathrm{~s}$ as a consequence of unfavourable flight speed histories. Whereas the leading B747 


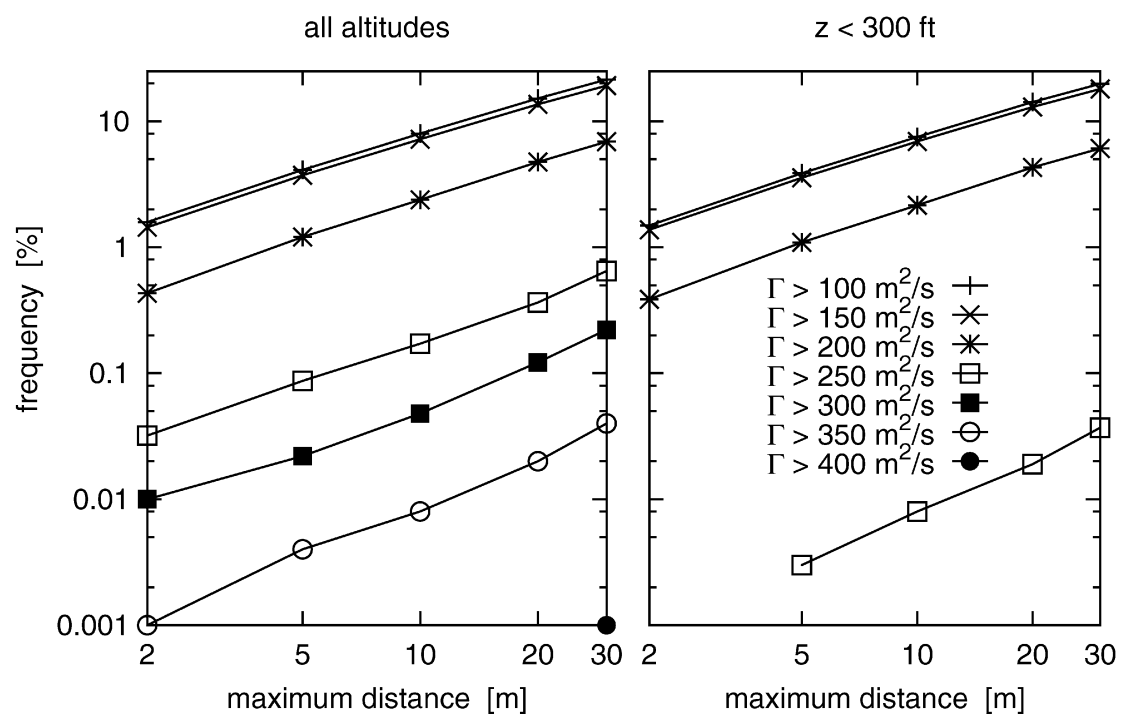

Fig. 8. Encounter frequency dependent on maximum aircraft distance to the vortex and minimum circulation.

flies one standard deviation of the average glide path deviations above the ILS, the A320 follows three standard deviations below the ILS. Due to a stably stratified atmosphere and weak wind shear the vortices have descended within that $100 \mathrm{~s}$ by only $100 \mathrm{~m}$ and are only laterally displaced from the ILS by $60 \mathrm{~m}$. The vortices are still very strong; rapid vortex decay commences only right after the encounter. This example illustrates that such an extreme event can only result from a combination of several adverse parameters.

The described statistics suggest that landing within an environment of more or less decayed wake vortices is daily practice. Three facts appear substantial to explain why current procedures are safe nevertheless. First, the area of a wake vortex which may exert hazardous forces and moments is relatively small. Second, the updrafts on both sides of the vortices are effective as protection by deflecting the encountering aircraft away from the most hazardous vortex cores. The first two factors can be quantified with VESA. Third, we presume that vortex deformation, which in general close to the ground starts to develop immediately after vortex generation, modifies the encounter geometry and normally reduces the impact time of adverse forces and moments such that encounters result in nonhazardous short-time interferences [23]. As reported by some of the authors aircraft performing sudden roll deflections prior to touch down can frequently be observed at busy airports. If the third hypothesis turns out to be correct it is clear that a model for vortex distortion must be integrated into risk assessment methodologies to meet absolute risk probabilities as required by ESARR 4 [7]. Relative comparisons of associated risks between a WVAS and an approved reference procedure (ICAO) are obviously feasible without consideration of vortex distortion provided that the distortion can be considered as similar for both procedures.

Although the above application example constitutes not a validated result it aligns nicely with reports of wake-vortex encounters. For instance, the sharply differentiated height range below $300 \mathrm{ft}$ in Fig. 7a corresponds exactly to the height range in which a pronounced accumulation of wake-vortex related incidents is reported on approaches to London Heathrow airport [3].

\section{Further development}

Comprehensive further developments of WakeScene are envisaged in order to make it more realistic and applicable to further scenarios. Among these are the extension of WakeScene to closely-spaced parallel runway systems within the DLR project Wirbelschleppe and to departure within the EU-project CREDOS [2]. It is foreseen to establish a consistent aircraft trajectory generator model which provides realistic flight path deviations in space and time and also takes into account the current wind and turbulence. The simplified hazard area prediction method (SHAPe) [27] will considerably refine the currently used area of interest by a hazard area which is based on a given roll control limit needed for compensation of imposed rolling moments for individual follower aircraft types. Outside this hazard area safe and undisturbed operations are assured. Direct coupling of WakeScene and VESA is intended for more flexibility. Finally, the development and integration of a parametric model for wake vortex deformation is advanced which will come along with the planned extension of VESA to threedimensional vortex structures.

\section{Conclusion}

WakeScene, a software package to determine wake vortex encounter probabilities is described. The severity of encounters identified by WakeScene can subsequently be evaluated with VESA (Vortex Encounter Severity Assessment). The components of WakeScene which model traffic mix, aircraft trajectories, meteorological conditions, wake vortex evolution, and potential hazard area are described in some detail together with the so far accomplished validation work. The methodology has 
been demonstrated for approach and landing but it can well be extended to other flight phases.

A particular strength of WakeScene is the comprehensive realistic meteorological data base for the Frankfurt terminal environment. The effort to establish a one-year synthetic meteorological data base is justified by (i) the dominant impact of meteorological conditions on wake vortex behaviour, (ii) the variety of parameter combinations observed in the planetary boundary layer which leads to a significant manifold of vortex behaviour scenarios, (iii) and the plausible assumption that safety-relevant situations typically arise from rare parameter combinations. Also within its other components WakeScene allows to stochastically vary relevant parameters or even to choose between different wake-vortex models.

A Monte-Carlo simulation with a sample size of 100,000 approaches of a heavy aircraft followed by medium weight class aircraft illustrates that the developed methodology yields reasonable, albeit not validated results. The simulation confirms the observation that most encounters occur at an altitude below $300 \mathrm{ft}$ above ground [3] whereas the strongest encounters only arise at higher altitudes. In ground proximity vortex rebound increases the encounter frequency but, on the other hand, encounter severity is limited by reliable vortex decay rates which are not always met aloft [19]. Severe encounters result from improbable combinations of several adverse parameters.

The frequency of encounters indicates that landing within an environment of more or less decayed wake vortices is daily practice. Decades of accident-free operations demonstrate that current procedures are safe nevertheless. We assume that inherent vortex deformation is decisive for the safety of current aircraft separations. The thus modified encounter geometry and reduced impact times of aerodynamic forces play down encounters to non-hazardous short-time interferences. Short-term roll of aircraft just before landing can quite often be observed at busy airports. In order to meet absolute risk probabilities as required by ESARR 4 [7] it is clear that vortex deformation must be considered in risk assessment methodologies. Relative risk assessments that refer to approved operations are feasible without consideration of vortex distortion provided that the distortion can be considered as similar for both procedures.

WakeScene simulation runs further demonstrate that the encounter probability may differ significantly for wake generators representing the respective lower and upper tails of the heavy weight class. This finding clearly indicates that considerable capacity gains could be achieved by simply increasing the number of aircraft weight class categories.

Any software which may be employed to prove the safety of a wake-vortex advisory system must constitute a sufficiently accurate representation of the projected operation and must be able to estimate the associated risk level. Therefore, appropriate validation of risk assessment tools is mandatory. However, for complete wake vortex risk assessment tools straightforward validation appears not feasible, because the significant manifold of modelled parameters can not be measured simultaneously and reconstructed consistently in a simulation. Nonetheless, systematic validation of complex assessment tools as WakeScene is possible for sub-models. For example, for the wake-vortex model and the meteorological data base comprehensive validations against measurement data are available. Assessments based on expert opinion, comparative studies, and different submodels can be used to provide further confidence in the suitability of the software package.

\section{References}

[1] Challenges to Growth 2004 Report, Eurocontrol, Brussels, 2004.

[2] CREDOS, http://www.eurocontrol.int/eec/credos/.

[3] J.B. Critchley, P.B. Foot, UK CAA wake vortex database: Analysis of incidents reported between 1982 and 1990, Civil Aviation Authority, CAA Paper 91015, 1991.

[4] A.C. de Bruin, L.J.P. Speijker, H. Moet, B. Krag, R. Luckner, S. Mason, S-wake - assessment of wake vortex safety, Publishable Summary Report, National Aerospace Laboratory, NLR-TP-2003-243, Amsterdam, 2003.

[5] G. Doms, U. Schaettler, The nonhydrostatic limited area model LM (Lokal Model) of DWD. Part I: Scientific documentation, Deutscher Wetterdienst, Offenbach, 1999.

[6] EUROCONTROL BADA Aircraft Performance Database, http://www. eurocontrol.fr/projects/bada/.

[7] Eurocontrol Safety Regulatory Requirement - ESARR 4, Risk Assessment and Mitigation in ATM, Eurocontrol, 2001.

[8] H. Frauenkron, M. Maiss, P. Nalpanis, FLIP - Flight Performance using Frankfurt ILS, DFS German Air Navigation Services, Air Traffic Management Division, Version 2.0, 2001.

[9] M. Frech, F. Holzäpfel, Skill of an aircraft wake-vortex model using weather prediction and observation, J. Aircraft 45 (2008) 461-470.

[10] M. Frech, F. Holzäpfel, A. Tafferner, T. Gerz, High-resolution weather data base for the terminal area of Frankfurt Airport, J. Appl. Meteor. Climat. 46 (2007) 1913-1932.

[11] T. Gerz, F. Holzäpfel, W. Bryant, F. Köpp, M. Frech, A. Tafferner, G. Winckelmans, Research towards a wake-vortex advisory system for optimal aircraft spacing, Comptes Rendus Physique 6 (2005) 501-523.

[12] G.A. Grell, S. Emeis, W.R. Stockwell, T. Schoenemeyer, T. Forkel, J. Michalakes, R. Knoche, W. Seidl, Application of a multiscale, coupled MM5/chemistry model to the complex terrain of the VOTALP valley campaign, Atmosph. Environ. 34 (2000) 1435-1453.

[13] J.N. Hallock, G.C. Greene, D.C. Burnham, Wake vortex research - a retrospective look, Air Traffic Control Quart. 6 (1998) 161-178.

[14] G. Höhne, R. Luckner, M. Fuhrmann, Critical wake vortex encounter scenarios, Aerosp. Sci. Technol. 8 (2004) 689-701.

[15] F. Holzäpfel, Probabilistic two-phase wake vortex decay and transport model, J. Aircraft 40 (2003) 323-331.

[16] F. Holzäpfel, T. Hofbauer, D. Darracq, H. Moet, F. Garnier, C. Ferreira Gago, Analysis of wake vortex decay mechanisms in the atmosphere, Aerosp. Sci. Technol. 7 (2003) 263-275.

[17] F. Holzäpfel, R.E. Robins, Probabilistic two-phase aircraft wake-vortex model: Application and assessment, J. Aircraft 41 (2004) 1117-1126.

[18] F. Holzäpfel, Probabilistic two-phase aircraft wake-vortex model: Further development and assessment, J. Aircraft 43 (2006) 700-708.

[19] F. Holzäpfel, M. Steen, Aircraft wake-vortex evolution in ground proximity: Analysis and parameterization, AIAA J. 45 (2007) 218-227.

[20] ICAO, Procedures for air navigation services - air traffic management, Doc 4444, ATM/501, 2001.

[21] H.-D. Joos, J. Bals, G. Looye, K. Schnepper, A. Varga, A multi-objective optimisation based software environment for control systems design, in: Proc. of 2002 IEEE International Conference on Control Applications and International Symposium on Computer Aided Control Systems Design, CCA/CACSD, Glasgow, Scotland, UK, 2002.

[22] B. Korn, A. Kuenz, 4D FMS for increasing efficiency of TMA operations, in: 25th Digital Avionics Systems Conference, Portland, OR, 2006, pp. 1E4-1-1E4-8.

[23] R.E. Loucel, J.D. Crouch, Flight-simulator study of airplane encounters with perturbed trailing vortices, J. Aircraft 42 (2005) 924-931.

[24] R. Luckner, G. Höhne, M. Fuhrmann, Hazard criteria for wake vortex encounters during approach, Aerosp. Sci. Technol. 8 (2004) 673-687. 
[25] R.E. Robins, D.P. Delisi, NWRA AVOSS wake vortex prediction algorithm version 3.1.1, NASA/CR-2002-211746, 2002, and NWRA-CR-00R229A.

[26] T. Sarpkaya, R.E. Robins, D.P. Delisi, Wake-vortex eddy-dissipation model predictions compared with observations, J. Aircraft 38 (2001) 687 692.

[27] C.W. Schwarz, K.-U. Hahn, Full-flight simulator study for wake vortex hazard area investigation, Aerosp. Sci. Technol. 10 (2006) 136-143.

[28] L.J.P. Speijker, J. Kos, H.A.P. Blom, G.B. van Baren, Probabilistic wake vortex safety assessment to evaluate separation distances for ATM operations, in: NLR TP 2000 326, 22nd ICAS 2000, Harrogate UK, 2000.
[29] L.J.P. Speijker, A. Vidal, F. Barbaresco, M. Frech, H. Barny, G. Winckelmans, ATC-wake: Integrated wake vortex safety \& capacity system, J. Air Traffic Control 49 (2007) 17-32.

[30] Wake Vortex Research Needs for "Improved wake vortex separation ruling" and "Reduced Wake Signatures", Final Report of the Thematik Network 'WakeNet2-Europe', in: 6th Framework Programme, National Aerospace Laboratory, NLR-CR-2006-171, Amsterdam, 2006.

[31] Y. Xie, J. Shortle, P. Choroba, Quantitative estimation of wake vortex safety using the P2P model, in: 6th USA/Europe Seminar on Air Traffic Management Research and Development, Baltimore, MD, 2005. 